\title{
Leading Conversations about Microaggressions, Bias, and Other Difficult Topics
}

\author{
Colleen M. Lewis \\ Computer Science Dept. \\ Harvey Mudd College \\ Claremont, CA, USA \\ lewis@cs.hmc.edu
}

\author{
Wendy M. DuBow \\ NCWIT \\ University of Colorado, Boulder \\ Boulder, CO, USA \\ wendy.dubow@ colorado.edu
}

\author{
Kyla McMullen \\ Comp. \& Info. Science \& Eng. \\ University of Florida \\ Gainesville, FL USA \\ drkyla@ufl.edu
}

\begin{abstract}
Many SIGCSE attendees are committed to inclusive teaching practices and creating an inclusive culture within their classrooms; yet, advocating for and sustaining these initiatives may require having difficult conversations with our colleagues and students. Understandably, many faculty are unsure about how to talk about sensitive topics such as race and gender with their colleagues and students. Research suggests that practicing some of these difficult conversations is essential to achieve the goals of inclusive teaching and culture. In our well attended session at SIGCSE in 2018, attendees learned strategies for responding to bias in academic settings. This was facilitated by playing two rounds of a research-based game developed by the NSF project CSTeachingTips.org (\#1339404). This session will extend the work begun last year by helping attendees to replicate this activity with their colleagues. In this special session, attendees will first play the game to practice those strategies in small groups and will then receive facilitation tips and guidance for conducting this activity on their own. All attendees will receive a printed copy of the game and a link to download and print more copies.
\end{abstract}

\section{CCS CONCEPTS}

- Social and professional topics Computing education

\section{KEYWORDS}

Microaggressions; bias; inclusive teaching

\section{SUMMARY AND OBJECTIVE}

The special session seeks to help attendees build their capacity to address and respond to bias in their academic environments. Most importantly, the special session will prepare attendees to replicate this session at their institution. Helping attendees bring learning from SIGCSE back to their colleagues is an important way to disseminate and scale strategies for creating more

Permission to make digital or hard copies of part or all of this work for personal or classroom use is granted without fee provided that copies are not made or distributed for profit or commercial advantage and that copies bear this notice and the full citation on the first page. Copyrights for third-party components of this work must be honored. For all other uses, contact the owner/author(s).

SIGCSE '19, February 27-March 2, 2019, Minneapolis, MN, USA

(C) 2019 Copyright is held by the owner/author(s).

ACM ISBN 978-1-4503-5890-3/19/02.

https://doi.org/10.1145/3287324.3287518 inclusive CS classrooms, ultimately broadening participation in computing.

\section{OUTLINE OF ACTIVITIES}

As attendees arrive, they will be asked to create groups of three to six people, which was successful in our SIGCSE Special Session in 2018 that was attended by approximately 80 people.

\subsection{Presentation of Session Goals (5 minutes)}

The session will begin with a brief introduction by the presenters to the structure and goals of the session.

Session Goals:

- Develop and practice strategies for responding to bias or problematic statements in the classroom.

- Practice explaining the benefits of inclusive teaching strategies to faculty and students who may be skeptical.

- Create connections among SIGCSE attendees committed to inclusive teaching practices.

- Prepare attendees to facilitate these discussion about bias with their colleagues.

After reviewing the goals for the session, the presenters will explain the first interactive portion of the special session. Groups will be provided a printed copy of the instructions and these instructions will be displayed on the screen for easy reference.

\subsection{Interactive Portion 1 (25 minutes)}

Groups will be asked to do a quick round of introductions before beginning the first interactive portion of the game. Attendees will then participate in the activity. In addition to the instructions, each group will have a set of sixteen cards. One person draws a card and read the question to a small group. Each member of the group answers the question or passes. After 3 minutes or hearing all answers, the person who read the question reads the sample answer from the card aloud and picks their favorite answer (the "winner"). The "winner" keeps the card to tally who won the most times. The person who reads the question rotates clockwise.

The cards encourage participants to (1) identify opportunities to apply inclusive teaching practices and (2) practice strategies for responding to bias or problematic statements in the classroom. The following list is a sample of the questions, which all begin "What would you do if..."

- students never ask questions during class? 
- during class a student with a lot of prior CS experience asks questions about advanced topics irrelevant to the class.

- some students did very poorly on a recent exam?

- your colleague says: "we want diversity, but we don't want to water down the content."

- your colleague says: "students should just tell me if I offend them."

- your colleague says: "women just aren't interested in CS."

- your colleague says: "there are so few Black and Latinx students in CS, it is a lot easier for them to get CS jobs."

- your colleague says: "Brenda needs to be more confident." Groups will vary in the speed that they progress through each of the cards, and we expect few groups to get through more than seven or eight cards. The goal is to have experience with the game before discussing how to facilitate this game with their colleagues.

The attendees may all have experience with these scenarios, and they can learn from each other to better be able to promote an inclusive classroom community.

\subsection{Synthesis of Strategies for Fostering Inclusion (20 minutes)}

After the game, the presenters will facilitate a debrief of the game identifying the key, relevant strategies for addressing these real-life scenarios and creating inclusive cultures. This debrief is focused on a list of four strategies for fostering inclusion that are listed with the game instructions:

- Empathize: Assume that there is a reason for students' behavior when they seem unengaged or are pursuing ineffective learning strategies.

- Promote belonging: Respond to behavior that may unintentionally signal to students that they don't belong in CS. This may be particularly important for students with less experience or who do not identify with CS stereotypes.

- Challenge stereotypes: Stereotypes can have negative consequences. Even seemingly positive stereotypes, like "Asians are good at math," are still problematic because they ignore individual variation, create unfair expectations, and may implicitly insult another group of people.

- Change structures: Sometimes initiatives to promote inclusion in CS focus on strategies for how students can respond to bias that they face. While these may be helpful strategies, they may unintentionally send the message that the student is as fault. Instead, we should focus on changing the structures that lead to bias or marginalization.

After reviewing the four strategies, the presenters will answer attendee questions to wrap up the debrief.

\subsection{Facilitation Tips and Discussion (25 minutes)}

For the final part of this session, the presenters will share recommendations for facilitating this activity. To do so, we will draw from materials we have developed in prior work training participants to be "ambassadors" for change in their home institutions. Small groups will then brainstorm and practice how they might propose this session with their colleagues and address any challenges they might face in facilitating the session. The ultimate goal is not to prepare participants to be "experts" but rather to be the "best prepared student in the class," facilitating these conversations back home in ways where everyone is learning together.

We will bring enough sets of cards for each attendee to have a copy. This will allow everyone who wants a set to have one and some attendees to be able to take multiple sets home or request additional sets be mailed to them.

\section{PRESENTERS}

Colleen Lewis is an assistant professor of CS at Harvey Mudd College where typically $40 \%$ to $50 \%$ of the CS majors identify as women. Colleen Lewis researches how people learn CS and how people feel about learning CS. Her research seeks to identify effective teaching practices for creating equitable learning spaces where all students have the opportunity to learn. Lewis is the PI for CSTeachingTips.org, a NSF-sponsored project (\#1339404) for disseminating effective CS teaching practices.

Wendy DuBow is Director of Evaluation and a Senior Research Scientist with the National Center for Women \& Information Technology (NCWIT) at the University of Colorado Boulder. Her research focuses on issues related to gender, diversity, and technology; organizational change and curriculum reform; and the interactions of majority and minority groups. She conducts mixed methods social science research, creates practical print and multimedia resources, and evaluates the effectiveness of the various programs and materials NCWIT produces.

Kyla McMullen is an Assistant Professor of Computer and Information Science and Engineering at the University of Florida. Dr. McMullen is the leader of the SoundPAD Laboratory at the University of Florida, which focuses on the Perception, Application, and Development of 3D audio in various contexts. Dr. McMullen also researches programming self-efficacy as an effect of IDE design, paired-programming, and active learning.

\section{SUITABILITY FOR A SPECIAL SESSION}

This innovative and interactive format would only be possible as a special session. This special session could be particularly helpful for new attendees so that they can meet other attendees. The session provides the important opportunity for attendees to become better prepared to facilitate conversations with their colleagues about microaggressions, bias, and other nonsense.

\section{ACKNOWLEDGEMENTS}

The development of CSTeachingTips.org and the special session content were funded by NSF grant \#1339404. These materials are based upon resources from NCWIT.org (NSF \#1725018). 